Proceedings of The

IEEE International Conference

on Industrial Technology, 1996

\title{
Applications of High Frequency Magnetic Components for Switching Resonant Mode Power Supply
}

\author{
F.K.Wong, J. W. Lu, H.B.Harrison and S.Dimitrijev \\ School of Microelectronic Engineering, Faculty of SCT, \\ Griffith University, QLD 4111, AUSTRALIA
}

\begin{abstract}
This paper presents the results of investigations on three types of high frequency transformers with different magnetic and winding configurations for the switching resonant mode power supply. The experimental results demonstrate the characteristics of self inductance and voltage ratio versus frequency. The magnetic flux and eddy-current distributions of Pot core, Planar core and Multi-element core transformers are calculated by a CAD/CAE software package which employs boundary element method technique. Both experimental and computer modelling results are used for optimum design of high frequency transformers and minimising the copper losses in transformer windings.
\end{abstract}

\section{INTRODUCTION}

One of the most volume-intensive components in switching converters is magnetic component. The size of this magnetic component can be decreased by operating the power converter at high frequencies. High frequency (HF) transformers are an indispensable part of most $\mathrm{HF}$ switching power supplies. However, they are not commercially available in a wide range of properties but are usually designed and constructed for the particular application. Although magnetic ferrites and power cores have improved in the past, transformers and inductors are a major obstacle to very-high-frequency operation. Parasitic elements, such as leakage inductance, winding capacitance, and skin and proximity effects, make it very difficult to design highfrequency magnetics [1] [2].

This paper presents the results of investigations on three types of HF transformer designs for the switching resonant mode power supply. Since HF magnetic ferrite materials have very low eddy-current loss, and have only hysteresis loss, the major concern is the copper loss in HF transformer. Therefore, the assumptions considered include a linear material and no hysteresis effects and thus it is not necessary to characterise the nature of the input voltage. A commercial software package OERSTED 2D/RS - Eddy Current Solver which employs the boundary element method is used to obtain the numerical results, such as magnetic flux and eddy-current for different winding configurations and help the designer/user to make an optimum design for the high frequency transformer.

\section{ANALYSIS OF POT CORE TRANSFORMER}

Pot core is mostly used as a HF inductor or transformer at low power levels $2100 \mathrm{w}$ in the switching power supplies.
Its major advantage is that the winding on the bobbin around the center post is almost entirely enclosed by ferrite material. This decreases its radiating magnetic field and hence is used when EMI/RFI problems must be minimised. Unfortunately, the lack of airflow around the windings causes them to operate at a higher temperature[3].

\section{A. Pot Core Winding Configurations}

TDK H6F pot core transformer with two different winding configurations is used as investigating transformer as shown in Fig. 1. PCT(I) is wounded on the separated primary and secondary winding sections of the bobbin without interweaving. PCT(II) is wounded on two sections with primary and secondary layer interweaving.



(a)

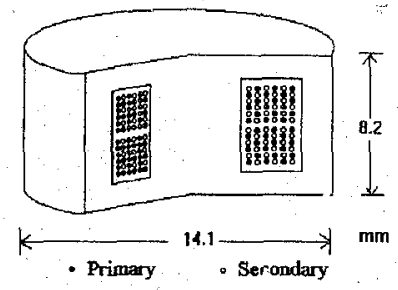

(b)
Fig: 1 Pot core winding configurations

(a) PCT(I) magnetic and separated windings structure,

(b) PCT(II) magnetic and winding structure with interweaving.

\section{B. Inductance vs Frequency Characteristics}

Figure 2 shows the experimental results for the inductance vs frequency characteristics with two differem winding configurations.

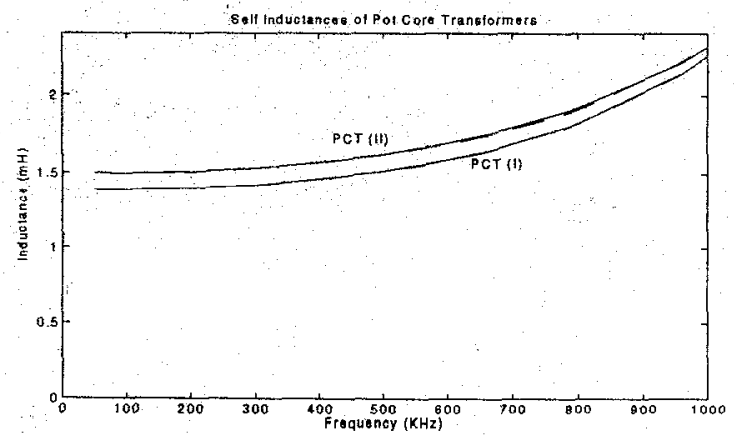

Fig. 2 Inductance vs frequency characteristics using TDK H6F pot core. 
A HP 4285A precision LCR meter was used to obtain results over a frequency range of $50 \mathrm{KHz}$ to $1 \mathrm{MHz}$. The inductance $\mathrm{L}$ increases with increasing frequency due to frequency characteristics of the TDK H6F ferrite material.

\section{Voltage Ratio vs Frequency Characteristics}

The input and output voltage ratio versus frequency characteristics for both windings at the no-load case are investigated. The voltage ratio for pot core structures shown in Fig. 3 demonstrates a frequency independent characteristics, which indicates that magnetic coupling is quite good at whole frequency range and the voltage ratio is almost equal to the winding ratio that is $\mathrm{N}_{1} / \mathrm{N}_{2}=\mathrm{V}_{1} / \mathrm{N}_{2}$.

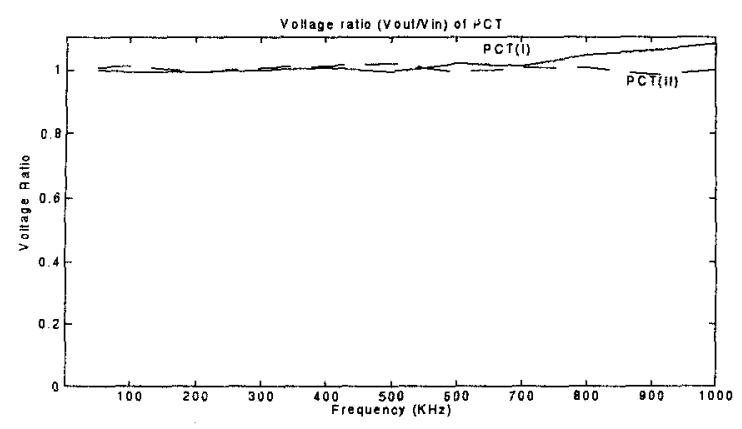

Fig. 3 Voltage ratio versus frequency characteristics

\section{Distribution of Magnetic Flux at $1 \mathrm{MHz}$}

The time harmonic magnetic field distribution within the core and winding was calculated by using OERSTED 2D/RS - Eddy Current Solver. Fig.4 and Fig.5 show the computed results for magnetic flux under the condition that the primary winding is excited and the secondary winding is open circuited. PCT(I) has an unbalanced flux distribution. In contrast with PCT(II), it has a uniform flux distribution.

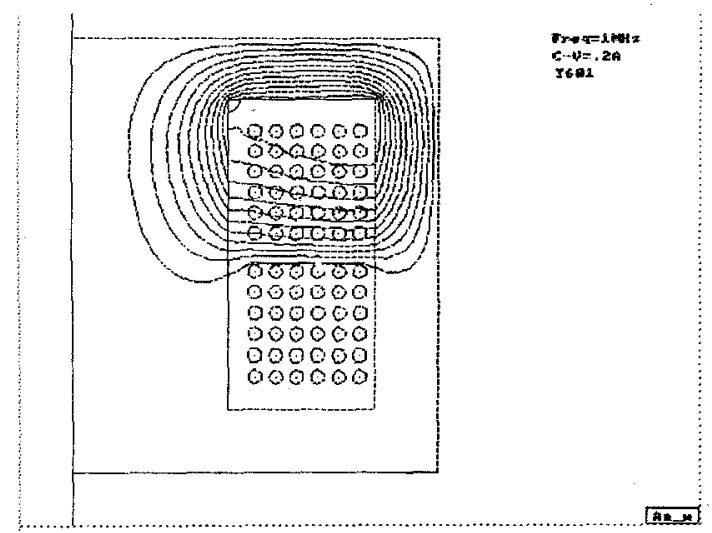

Fig. 4 Distribution of magnetic flux in PCT(D), $\mu=800$.



Fig. 5 Distribution of magnetic flux in PCT(II), $\mu=800$.

\section{ANALYSIS OF PLANAR CORE TRANSFORMER}

Advantages of planar core transformer designs include a low profile, good heat transfer properties, and operation at high power densities because of low power losses. These advantages are important if one is to realise a reduction in the size of magnetic components in high frequency converters. Also, lack of accurate data on parasitic elements, such as leakage inductance, winding capacitance, and skin and proximity effect makes it very difficult to design high-frequency magnetic devices correctly at the first time. Several planar inductive components with different configurations have been reported, for instance the Sandwich Transformer [4], the Matrix Transformer [5], the Thin Film Microtransformer with Circular Spiral Coils [6] and Toroidal-Meander Type Integrated Inductor with a Multilevel Meander Magnetic Core [7].

\section{A. Planar Core Winding Configurations}

DC-DC converters operating at high frequencies over $1 \mathrm{MHz}$ require inductance values in the range of a few micro-henries. Sandwich transformers using an open magnetic structure and a spiral or meander type winding configurations are shown in Fig. 6.

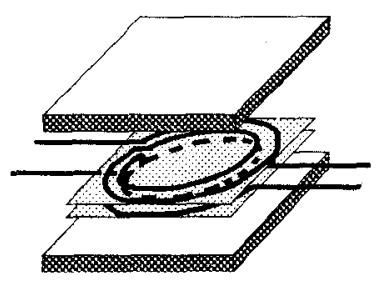

(a)

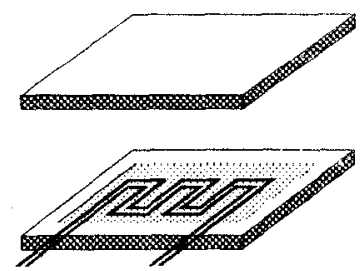

(b)
Fig. 6 Planar sandwich spiral and meander winding configurations with $17.6 \mathrm{~mm} \times 17.6 \mathrm{~mm} \times 2.0 \mathrm{~mm}$ magnetic ferrite (a) PST(I) is a spiral type winding structure $\left(\mathrm{N}_{1}=\mathrm{N}_{2}=3\right)$, (b) PST(II) is a meander type winding structure. 


\section{B. Inductance vs Frequency Characteristics}

Figure 7 presents the experimental results for the inductance vs frequency characteristics of a planar magnetic transformer with a spiral type and meander type winding configurations. The results are obtained over a frequency range of $50 \mathrm{KHz}$ to $1 \mathrm{MHz}$. The inductance $\mathrm{L}$ decreases with increasing frequency, while the meander type winding remains constant for whole frequency range. The inductance value of secondary winding of PST(II) is lower than primary one because the length of secondary winding is shorter than primary winding.

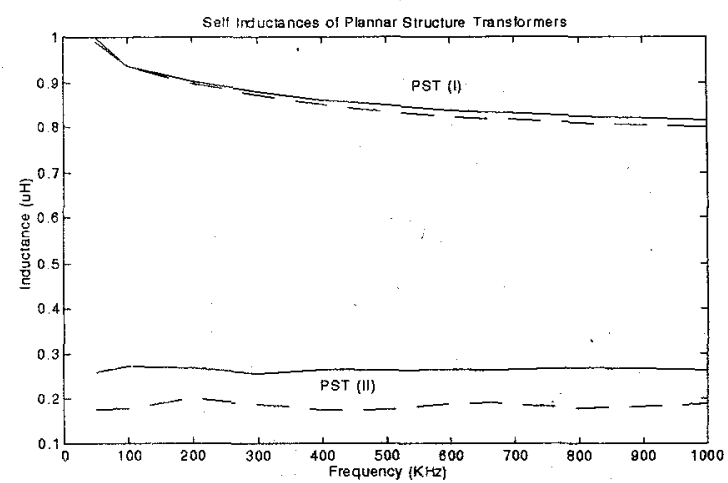

Fig. 7 Self inductance vs frequency characteristics, where - and - indicate the results for primary and secondary windings respectively.

\section{Voltage Ratio vs Frequency Characteristics}

The voltage ratio vs frequency for open core planar magnetic structures illustrates a frequency dependent characteristic shown in Fig. 8. The result is similar to the results which have been obtained for the thin film microtransformer with closed magnetic core structure [6]. The measured results also indicate that the voltage ratio remains constant above $300 \mathrm{KHz}$. Magnetic coupling is smaller than pot core at whole frequency range and thus the voltage ratio is not equal to the winding ratio that is $\mathrm{N}_{1} / \mathrm{N}_{2} \neq \mathrm{V}_{1} / \mathrm{N}_{2}$

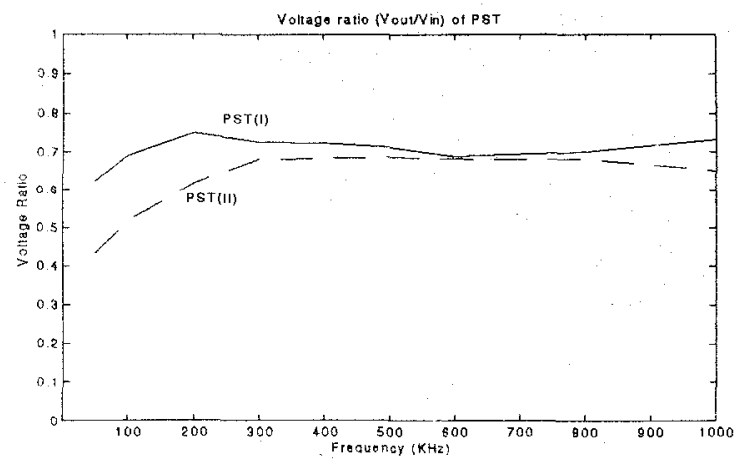

Fig. 8 PST(I) and PST(I) voltage ratio versus frequency characteristics.

\section{Distribution of Magnetic Flux at $1 \mathrm{MHz}$}

Figure 9 shows the computed results for magnetic flux under the condition that the primary winding is excited and the secondary winding is open circuited. The large air gap in this planar core transformer makes the magnetic system linear at the expenses of a reduction in the coupling efficiency and a lowery of the mutual inductance.

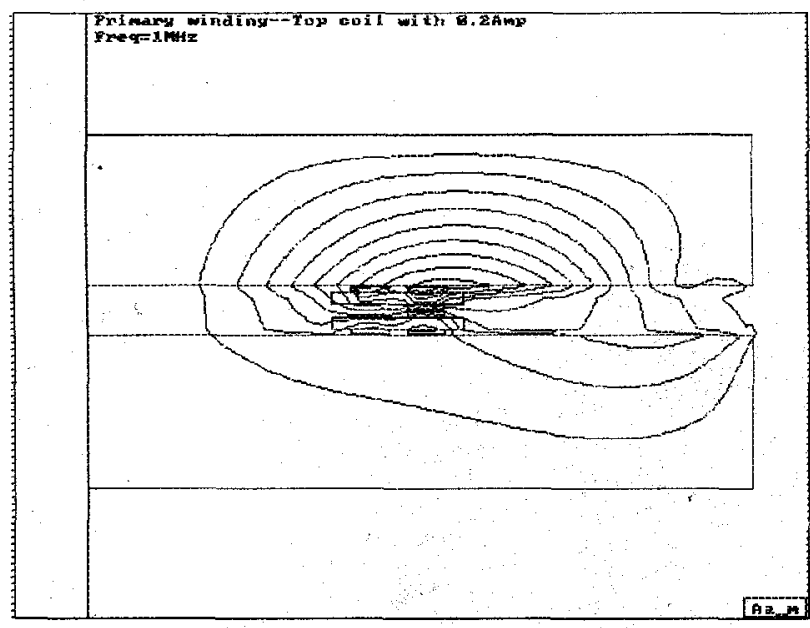

(a) Axi-symmetric cross section of PST(I) sparal type magnetic and winding configurations, $\mu=800$.



(b) Cross section of PST(II) meader type magnetic and winding configurations, $\mu=800$.

Fig.9 Distribution of magnetic flux in planar spiral and meander type transformers.

\section{ANALYSIS OF MULTI-ELEMENT TOROTDAL CORE TRANSFORMER}

Multi-element toroidal core transformer has been introduced to switching power supply recently [8] and it has been commonly used as a broadband RF transformer in communications systems[9]. The advantages of the multi- 
element transformer are the excellent symmetry results from the construction style and uniform distribution of magnetic flux and extremely low eddy-current density in the windings. The operation frequency can be uesd over a frequency range of $1 \mathrm{MHz}$ to $50 \mathrm{MHz}$.

\section{A. Multi-Element Toroidal Core Winding Configurations}

A small-diameter copper tube which is U-shaped, and over which several high- $\mu$ toroid cores have been placed. The ends of the tube are soldered onto the printed circuit board as shown in Fig.10. U-shaped copper tube has a 1 turn primary winding and an insulated hookup wire is passed through the tube and serves as the secondary winding of the transformer. The number of turns to be used will depend upon the transformation ratio needed.

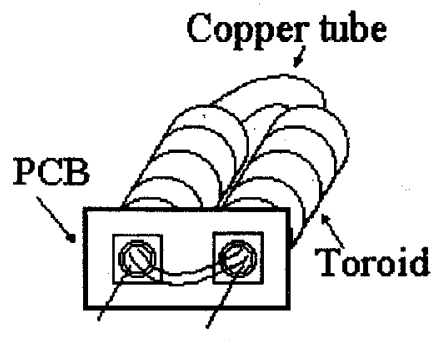

Winding

Fig. $10 \mathrm{U}$-shaped multi-element transformer

\section{B. Inductance vs Frequency Characteristics}

Figure 11 presents the experimental results for the self inductance vs frequency characteristics of the multi-element transformer over a frequency range of $50 \mathrm{KHz}$ to $1 \mathrm{MHz}$. The inductance $L$ remains constant for the whole frequency range. The inductance result for primary is quite lower due to 1 turn winding structure. The inductance of secondary winding is higher because of 8 turns in secondary.

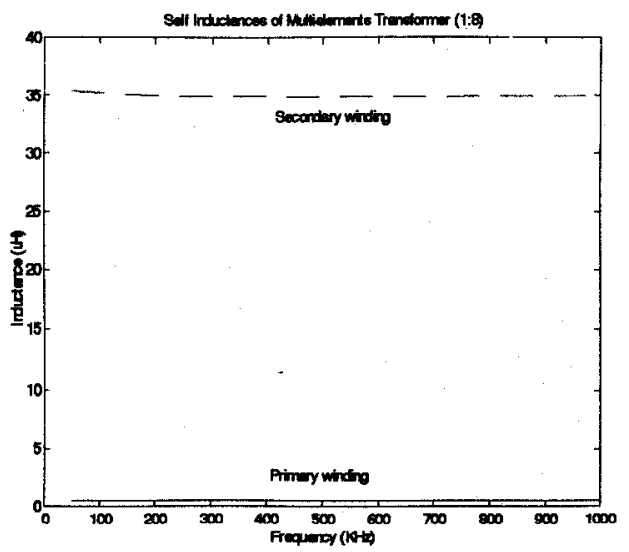

Fig. 11 Self inductance vs frequency characteristics, where - and - indicate the results for primary and secondary windings respectively.

\section{Voltage Ratio vs Frequency Characteristics}

Figure 12 shows the input and output voltage ratio versus frequency characteristics for the no-load case. The measured results indicate that magnetic coupling is smaller than pot core structure and thus the voltage ratio is less than the winding ratio that is $\mathrm{N}_{1} / \mathrm{N}_{2}>\mathrm{V}_{1} / \mathrm{N}_{2}$.

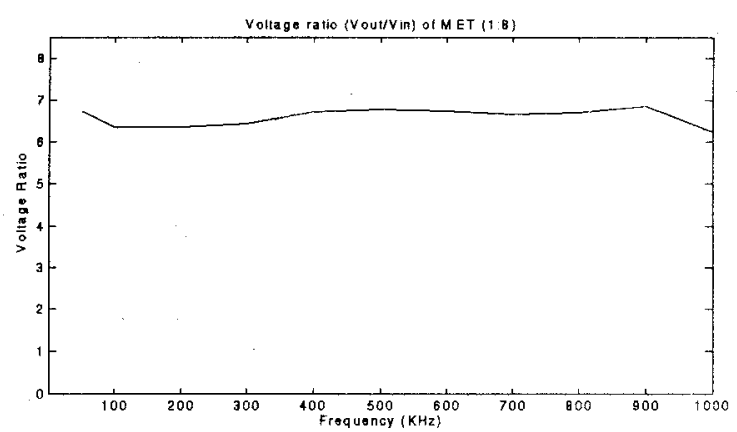

Fig. 12 Voltage ratio versus frequency characteristics

\section{Distribution of Magnetic Flux at $1 \mathrm{MHz}$}

The time harmonic magnetic field distribution within the core and winding was calculated by CAD/CAE tool. Fig.13 shows the computed results for magnetic flux under the condition that the primary winding is excited and the secondary winding is open circuited. The symmetrical and uniform flux distribution result presents an excellent transformer construction.

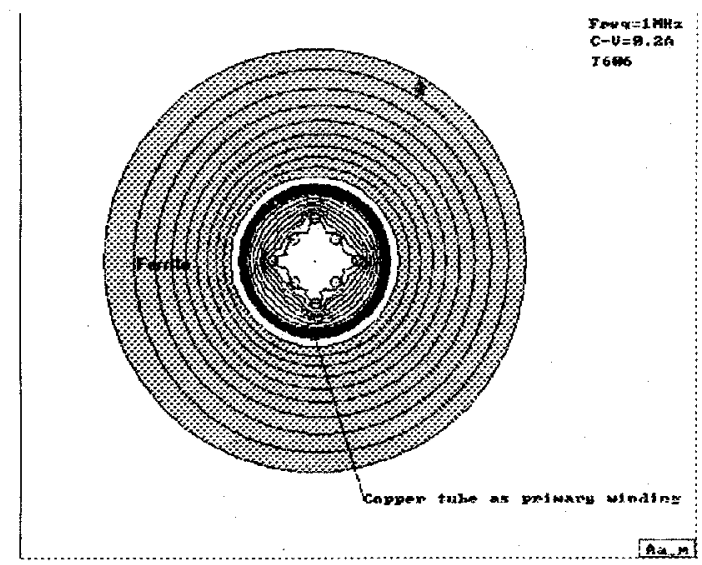

Fig. 13 Distribution of magnetic flux in multi-element transformer, $\mu=800$.

\section{COMPARISON OF HIGH FREQUENCY TRANSFORMERS}

Figure 14 shows the computer simulation results for maximum eddy-current density. From the computing results, we can find that multi-element core transformer has a minimum eddy-current density and the pot core transformer 
with separated winding configuration has a very high eddycurrent density.



Fig. 14 Maximum eddy-current density in HF transformers.

The comparison of three types of HF transformers has been made in Table 1. The results present the advantages and disadvantages of each transformer. The multi-element core transformer has a low copper loss at high operating frequency, it can be used for high frequency and high power application. The manufacture cost will be reduced by using multi-wire cable and printed circuit board with new assembly technology. The planar core transformer can be placed inside of power ICs for the low power application. The leakage inductance and copper loss can be significantly reduced by using new winding configuration (PCT(II)) as shown in Fig. 5 and Fig. 14 respectively.

Table 1. General comparison of HF Transformers.

\begin{tabular}{|c|c|c|c|}
\hline $\begin{array}{c}\text { Transformer } \\
\text { sale }\end{array}$ & MET & PCT & PST \\
\hline $\begin{array}{c}\text { Ferrits } \\
\text { Materal } \\
\text { Frequency }\end{array}$ & $\begin{array}{c}\text { Iron } \\
\text { Powder } \\
\text { (Philips) }\end{array}$ & $\begin{array}{c}\text { MnZn } \\
\text { (TDK) }\end{array}$ & $\begin{array}{c}\text { NiZn } \\
\text { (TDK) }\end{array}$ \\
\hline $\begin{array}{c}\text { Winding } \\
\text { Shieiding }\end{array}$ & Yes & $\leq 1 \mathrm{MHz}$ & $>1 \mathrm{MHz}$ \\
\hline Cone Cost & Low & High & Yes \\
\hline Manu, Cost & High & Medium & Low \\
\hline Copper Loss & Low & Medium & Low \\
\hline $\begin{array}{c}\text { Power } \\
\text { Density }\end{array}$ & High & Medium & Low \\
\hline
\end{tabular}

\section{CONCLUSIONS AND FUTURE WORK}

The paper presented the experimental anơ numerical results for three different types of HF transformers. The primary winding was subjecter to a $50 \mathrm{kHz}$ to $1 \mathrm{MHz}$ sinusoidal current source and the secondary winding was left open circuited. The inequality of planar core and multielement core between the secondary to primary voltage ratio and the turns ratio indicate that the magnetic coupling is smaller than pot core. The numerical results obtained by using a CAD/CAE tools which employs the boundary element method show that the distribution of magnetic flux and eddy-currents can be controlled by using proper winding structures. Future work will concentrate on optimizing the design of the magnetic system subject to constraints such as a desired magnetic coupling ratio, desired heat transfer materials, EMI and the calculation of losses which include hysterisis effects.

\section{ACKNOWLEDGMENT}

The authors wish to thank the Australia Research Council for supporting this research work.

\section{REFERENCES}

[1] J. G. Kassakian, M. F. Schlecht, "High-Frequency High Density Converters for Distributed Power Supply Systems," pp. 362-376, Proceedings of the IEEE, Vol. 76, No. 4, pp. 362-376, April 1988.

[2] Richard Farrington, Milan M. Jovanovic and Fred C. Lce, "Design Oriented Analysis of Reactive Power in Resonant Converters," IEEE TRANS. ON POWER ELECTRONICS, Vol .8, No.4, 1993.

[3] A. I. Pressman, Switchng Power Supply Design, McGraw-Hill, Inc. 1991

[4] J. Lu, F. Danson and S. Yamada, "Analysis of High Frequency Planar Sandwich Transformers for Switching Converters," IEEE TRANS. ON MAGNETICS, Vol. 31, NO. 6, NOV. 1995.

[5] Khai D. Ngo, E. Alpizar and J. Kenneth Watson, "Modeling of Magnetizing Inductance and Leakage Inductance in a Matrix Transformer;" IEEE TRANS. ON POWER ELECTRONICS, Vol .8, No.2, pp 200207, April 1993

[6] Kazuyuki Yamaguchi, Shigehiro Ohnuma, etc., "Characteristics of a Thin Film Microtransformer with Circular Spiral Coils," IEEE TRANS. ON MAGNETICS; Vol. 29, No.5, pp 2232-2237, Sep. 1993.

[7] Chong H, Ahn, and Mark G. Allen, "A New ToroidalMeander type Integrated Inductor with a Multilevel Meander Magnetic Core," IEEE TRANS. ON MAGNETICS, Vol. 30, No.1, pp 73-79, Jan. 1994

[8] R. Williams, D.A. Grant and J. Gowar, "Use of Multielement Transformers in Quasi-resonant Convertors," IEE Proceedings-B, Vol. 140, N0.6 Nov. 1993.

[9] W. Hayward and D. DeMaw, Solid State Design for the Radio Amateur, American Radio Relay League, Inc.1977. 